\title{
Topical NSAIDs, intravitreal dexamethasone and peribulbar triamcinolone for pseudophakic macular edema
}

\author{
Javier Obis* ${ }^{*}$, Luis Arias, Daniel Lorenzo, Noel Padron-Perez, Pere Garcia-Bru, Estefania Cobos,
} Rahul Morwani and Jose Caminal

\begin{abstract}
Background: The purpose of this study is to assess the effectiveness of topical nonsteroidal anti-inflammatory drugs (NSAIDs) and corticosteroids (intravitreal dexamethasone and peribulbar triamcinolone) in treating pseudophakic macular edema (PME).

Methods: Retrospective study of 33 eyes. Variables included best corrected visual acuity (BCVA; logMAR scale) and central retinal thickness (CRT) and central choroidal thickness (CCT) assessed with swept-source OCT. All patients were initially prescribed topical NSAIDs and reevaluated after 2 months. If improvement in BCVA or CRT was noted, topical NSAIDs were continued until resolution. If no improvement was observed at 2 months or subsequent visits, intravitreal dexamethasone implant was performed. Patients who refused intravitreal treatment were offered peribulbar triamcinolone.

Results: After treatment with topical NSAIDs for a median of 2 months, BCVA increased significantly from 0.5 to 0.3 while CRT decreased significantly from 435 to $316 \mu \mathrm{m}$. PME resolved in 19 of the 33 eyes (57.6\%). Of the 14 recalcitrant cases, 13 were treated with corticosteroids. Of these 13 cases, 9 (69.2\%) resolved. BCVA increased non-significantly from 0.7 to 0.4. CRT and CCT decreased significantly from 492 to $317 \mu \mathrm{m}$ and from 204 to $182 \mu \mathrm{m}$ respectively.

Conclusions: The overall success rate of the treatment algorithm was greater than $80 \%$, a remarkable finding considering that no randomized study has yet been conducted to determine the optimal therapeutic protocol for PME. This is the first study to evaluate choroidal thickness in PME using SS-OCT, which could play a key role in its pathophysiology and provide useful information to improve the management of PME.
\end{abstract}

Keywords: Pseudophakic macular edema / Irvine Gass syndrome, Topical NSAIDs, Intravitreal dexamethasone, Peribulbar triamcinolone, Central retinal thickness, Central choroidal thickness

\section{Background}

The term postsurgical macular edema is used to describe the macular edema that appears in some eyes after ocular surgery such as cataract surgery or vitrectomy, and it is an important cause of postsurgical vision loss. Macular

*Correspondence: luisarias@telefonica.net

Ophthalmology department, Bellvitge University Hospital, Carrer de la

Feixa Llarga, s/n, 08907. Hospitalet de Llobregat, Barcelona, Spain edema that develops after complicated or uncomplicated cataract surgery is known as pseudophakic macular edema (PME) or Irvine-Gass syndrome (IGS). PME was first described by Irvine in 1953 [1] and subsequently documented on angiography by Gass and Norton in 1966 $[2]$.

The reported incidence of PME detected with optical coherence tomography (OCT) ranges from 5.5 to $9 \%$ $[3,4]$. The incidence of clinically-significant PME, which permits use, sharing, adaptation, distribution and reproduction in any medium or format, as long as you give appropriate credit to the original author(s) and the source, provide a link to the Creative Commons licence, and indicate if changes were made. The images or other third party material in this article are included in the article's Creative Commons licence, unless indicated otherwise in a credit line to the material. If material is not included in the article's Creative Commons licence and your intended use is not permitted by statutory regulation or exceeds the permitted use, you will need to obtain permission directly from the copyright holder. To view a copy of this licence, visit http://creativecommons.org/licenses/by/4.0/. The Creative Commons Public Domain Dedication waiver (http://creativeco mmons.org/publicdomain/zero/1.0/) applies to the data made available in this article, unless otherwise stated in a credit line to the data. 
includes decreased visual acuity or metamorphopsia, ranges from 0.95 to $2.35 \%$ [5, 6]. PME typically develops from 4 to 12 weeks after the surgery, with a peak incidence around week 6 [7]. Several risk factors have been suggested, including intraoperative capsular rupture, epiretinal membrane, diabetes or uveitis $[4,6,7]$. Although the pathophysiology of PME is not well-understood, some reports suggest that it may be related to an inflammatory process that disrupts the blood-retina barrier, leading to increased vascular permeability and subsequent fluid accumulation $[7,8]$.

Although several different treatment options for PME have been investigated in recent years $[9,10]$, no randomized studies have been conducted to determine the optimal therapeutic protocol for PME. In routine clinical practice, topical nonsteroidal anti-inflammatory drugs (NSAIDs) are commonly used as first-line therapy, either alone or in combination with oral acetazolamide $[9,10]$. If first-line treatment is unsuccessful, a wide range of second-line treatments are available [11], most commonly corticosteroids such as subtenon or peribulbar triamcinolone [12] or intravitreal dexamethasone [11, 13-16]. Other second-line alternatives have been proposed, including intravitreal anti-VEGF injections (with conflicting results) $[17,18]$, or infliximab (which may be associated with retinal toxicity) $[19,20]$. Treatment selection is further complicated by the fact that PME (defined as macular edema after cataract surgery) and macular edema occurring after vitrectomy could be two different entities that respond differently to treatment [21]. Considering the aforementioned, more randomized controlled studies with larger number of patients would be necessary to evaluate the treatment of clinically significant PME.

In this context, the aim of the present study was to retrospectively assess the effectiveness of topical NSAIDs, intravitreal dexamethasone and peribulbar triamcinolone in patients with clinically-significant PME treated in a real-world clinical setting. In this case series, we evaluated best-corrected visual acuity (BCVA) and central retinal and choroidal thickness using swept-source OCT (SS-OCT). Finally, we describe a proposed treatment algorithm.

\section{Material and methods}

This was an observational, retrospective, consecutive case series involving patients treated at a tertiary care hospital (Bellvitge University Hospital in Barcelona, Spain). All the data were obtained from the medical records of patients with PME who had been previously treated following the treatment algorithm that will be explained below. Inclusion criteria were as follows: 1) cataract surgery (complicated or uncomplicated), 2) presence of symptomatic postoperative macular edema on SS-OCT (in all cases, visual acuity improved after cataract surgery but worsened afterwards). Exclusion criteria included a history of any of the following: diabetic retinopathy including diabetic macular edema, retinal vein occlusion, posterior uveitis, age-related macular disease (including presence of drusen), central serous chorioretinopathy, or any other condition that could potentially confound the diagnosis, affect retinal or choroidal thickness, or modify treatment response. In order to rule out these conditions, no patient with history of diabetes was included in this study, and all the patients underwent slit lamp examination, funduscopy and SS-OCT examination before cataract surgery.

BCVA was evaluated (logMAR scale) and SS-OCT was performed to assess central retinal thickness (CRT) and central choroidal thickness (CCT) at diagnosis of PME and thereafter every 2 months.

The Deep Range Imaging (DRI) Triton SS-OCT device (Topcon, Tokyo, Japan) was used to measure CRT and $\mathrm{CCT}$, and to determine the presence of intraretinal and/ or subretinal fluid. The DRI Triton SS-OCT utilizes a $1050 \mathrm{~nm}$ wavelength light, with 100,000 A-scans per second, and axial and transverse resolutions of 8 and $20 \mu \mathrm{m}$, respectively. We used the Radial protocol, which consists of a $12 \mathrm{~mm}$ radial scan of the macular area to obtain thickness measurements of the nine macular areas of the Early Treatment Diabetic Retinopathy Study (ETDRS). The retinal and choroidal values of the central ETDRS area were registered and included in our study. The DRI Triton SS-OCT yields a quality scale that ranges from 0 (lowest quality) to 100 (highest quality). We included only images whose score was $>50$.

The following clinical and demographic variables were registered: patient sex and age; duration of topical NSAIDs; number of corticosteroids injections; and time elapsed from surgery to diagnosis of PME.

Figure 1 shows the treatment algorithm applied, the number of patients who received each treatment, and the number of cases in which the edema resolved or persisted. In all cases, first-line treatment consisted of once-daily topical NSAIDs (nepafenac $3 \mathrm{mg} / \mathrm{ml}$; Nevanac, Novartis) administered for 2 months, after which the BCVA was evaluated, and the CCT and CRT were measured by SS-OCT.

In patients who showed improvement in BCVA or CRT decrease at the first follow-up visit (2 months after starting topical NSAIDs), topical NSAIDs were continued and a second follow-up was scheduled for 2 months later. At that second visit, if the edema had resolved (no intraretinal or subretinal fluid on SS-OCT), NSAIDs were discontinued and the patient was scheduled for another follow-up visit 2 months later to verify the continued 


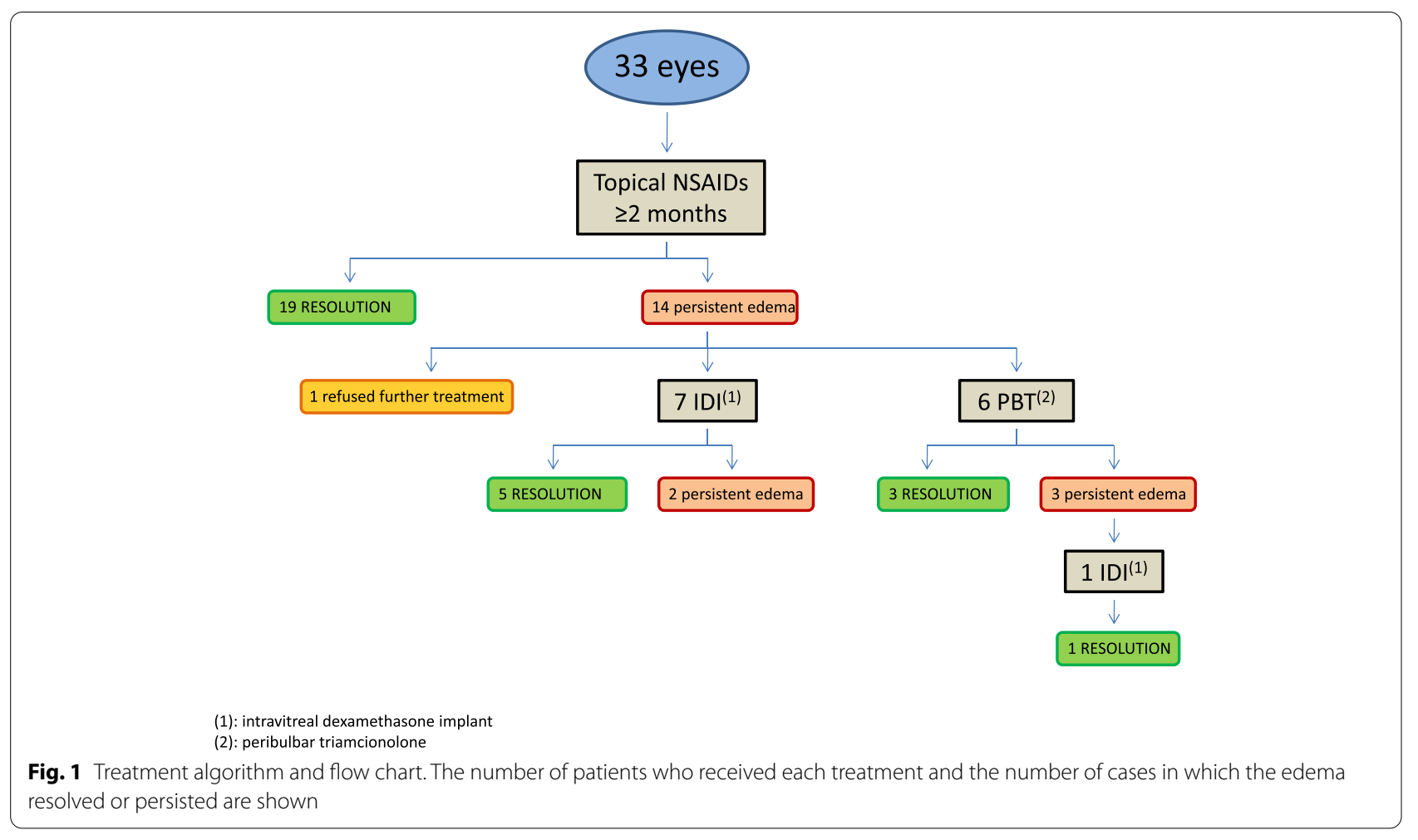

absence of the edema. If at any subsequent visit one patient showed improvement in BCVA or CRT decrease but the edema had not resolved completely, topical NSAIDs were continued and the patient was scheduled for another follow-up visit. Thus, topical NSAIDs were continued until complete resolution of the edema or until no response was observed at a follow-up visit.

At the first follow-up visit, patients who showed no response to the two-month course of topical NSAIDs (no improvement in BCVA and no decrease in CRT) were prescribed an intravitreal dexamethasone implant (Ozurdex, Allergan). Patients who exhibited an initial improvement in BCVA or CRT with topical NSAIDs at any follow-up visit but showed no improvement at a subsequent visit, were also prescribed intravitreal implant of dexamethasone.

After dexamethasone implant, follow-up visits were scheduled every 2 months. In patients in whom the edema decreased but failed to resolve completely (persistence of intraretinal or subretinal fluid on SS-OCT) at least 4 months after the first implant, a second dexamethasone implant was performed. In contrast, patients whose edema resolved (no intraretinal or subretinal fluid on SS-OCT) after the dexamethasone implant were followed for at least 6 months to verify no recurrence of the edema. Dexamethasone implants were discontinued when persistent absence of edema was verified (healed PME) or when no decrease of intraretinal or subretinal fluid was observed at all in the follow-up visits (refractory PME). Finally, patients who refused intravitreal treatment were offered peribulbar triamcinolone (Trigon, Bristol-Myers Squibb).

Written informed consent was deemed unnecessary as this was a retrospective study carried out in the context of routine clinical practice. All patient data were anonymized for this study. All confidential data are protected according national legislation. This study and manuscript have been approved by the Research Ethics Committee of Bellvitge University Hospital.

\section{Statistical analysis}

All variables were registered in a spreadsheet (Microsoft Excel). Statistical analysis was performed using the SPSS software program, v. 20.0 (SPSS, Inc., Chicago, IL). All variables showed a non-normal distribution, except for age (Shapiro-Wilk test). Consequently, the Wilcoxon test was used to compare the medians of the variables. Differences were considered statistically significant for $p \leq 0.05$. 


\section{Results}

Thirty-three eyes from 33 patients were included in the study. Table 1 shows baseline parameters. Mean patient age was 73.8 years (SD \pm 9.27 ). Twenty-three patients $(69.7 \%)$ were male. Of the 33 patients, $48.5 \%(n=16)$ were consequent to uncomplicated cataract surgery, and $51.5 \%(n=17)$ were consequent to complicated cataract surgery (posterior capsular rupture). The median time to diagnosis was 1 month. Mean follow-up was 9 months.

Median baseline values were as follows: BCVA, 0.5 logMAR range 0 [2-].1.30 (decimal scale 0.32 range 0.63-0.05); CRT, $435 \mu \mathrm{m}$ range 27 [2-]786 $\mu \mathrm{m}$; and CCT, $253 \mu \mathrm{m}$ range $79-358 \mu \mathrm{m}$. There were no significant differences in baseline parameters between patients who had undergone complicated vs. uncomplicated cataract surgery.

Table 2 shows the baseline and post-treatment (topical NSAIDs) results. Median BCVA improved to 0.3 (decimal scale 0.5); median CRT decreased to $316 \mu \mathrm{m}$; and median CCT decreased to $256 \mu \mathrm{m}$. Compared with the baseline values, significant differences (Wilcoxon signedrank test) were observed for BCVA $(-2.914, P=0.004)$ and CRT $(-2.963, P=0.003)$, but not for CCT $(-1.357$, $P=0.175)$.

In 19 eyes (57.6\%) the edema resolved with topical NSAIDs after a median of 2 months. In the 14 eyes (42.4\%) that failed to respond to topical NSAIDs, second-line corticosteroid treatment was proposed; however, one patient refused any further treatment and thus 13 patients $(39.4 \%)$ received second-line therapy. Of these, seven received intravitreal dexamethasone implant, which resolved the edema in five cases (71.4\%) after a median of 2 injections. The remaining six patients received peribulbar triamcinolone (because they preferred to avoid intravitreal injection), which resolved the edema in three cases $(50 \%)$ after a median of one injection. One of the patients with persistent macular edema after peribulbar triamcinolone agreed
Table 2 Characteristics of the 33 patients treated with NSAIDs

\begin{tabular}{llll}
\hline & Baseline & Final & $\boldsymbol{P}^{*}$ \\
\hline BCVA, logMAR (range) & $\mathbf{0 . 5}(0.2-1.30)$ & $\mathbf{0 . 3}(0.0-1.30)$ & $\mathbf{0 . 0 0 4}$ \\
CRT, $\mu$ m (range) & $\mathbf{4 3 5 ( 2 7 2 - 7 8 6 )}$ & $\mathbf{3 1 6 ( 2 2 7 - 8 7 1 )}$ & $\mathbf{0 . 0 0 3}$ \\
CCT, $\mu$ m (range) & $253(79-358)$ & $256(76-382)$ & 0.175 \\
Resolution of PME, n (\%) & $19 / 33(\mathbf{5 7 . 6 \% )}$ & & \\
$\begin{array}{l}\text { Duration of treatment, } \\
\text { months (range) }\end{array}$ & $2(2-9)$ & & \\
\hline
\end{tabular}

* Wilcoxon signed-rank test

Abbreviations: NSAIDs Non-steroidal anti-inflammatory drugs, logMAR Logarithm of the minimum angle of resolution, CRT Central retinal thickness, CCT Central choroidal thickness, PME Pseudophakic macular edema

to receive intravitreal dexamethasone, which resolved the edema after two injections. (Fig. 1)

Given the limited number of patients who received intravitreal dexamethasone implant and/or peribulbar triamcinolone $(n=13)$, we decided to group these patients into a single group (corticosteroid) for statistical analysis. Table 3 shows the baseline and post-treatment results. Nine of the 13 cases $(69.2 \%)$ resolved with second-line corticosteroid treatment. Median BCVA improved from 0.7 to 0.4 (decimal scale from 0.2 to 0.4 ), but the difference was not statistically significant (Wilcoxon signed-rank test $-1.577, P=0.115$ ); median CRT decreased significantly from 492 to $317 \mu \mathrm{m}$ $(-3.036, P=0.002)$; and median CCT decreased significantly from 204 to $182 \mu \mathrm{m}(-3.185, P=0.001)$.

Figure 2 shows the SS-OCT scans of a patient who required intravitreal dexamethasone after suboptimal response to topical NSAIDs.

No adverse treatment-related events were observed (keratitis or other ocular surface disturbances with NSAIDs; significant intraocular pressure elevation with corticosteroids; endophthalmitis with intravitreal dexamethasone implant).

Table 3 Characteristics of the 13 eyes treated with corticosteroids (IDI+PBT)

\begin{tabular}{llll}
\hline & Baseline & Final & $\boldsymbol{P}^{*}$ \\
\hline BCVA, logMAR (range) & $0.7(0.30-1.30)$ & $0.4(0.05-1.30)$ & 0.115 \\
CRT, $\mu$ m (range) & $\mathbf{4 9 2 ( 3 5 9 - 8 7 1 )}$ & $\mathbf{3 1 7}(202-625)$ & $\mathbf{0 . 0 0 2}$ \\
CCT, $\mu$ m (range) & $\mathbf{2 0 4}(76-382)$ & $\mathbf{1 8 2}(66-284)$ & $\mathbf{0 . 0 0 1}$ \\
Resolution of PME & $9 / 13(\mathbf{6 9 . 2} \%)$ & & \\
Number of injections & IDI: $2(1-5)$ & PBT: 1 (1-2) &
\end{tabular}

* Wilcoxon signed-rank test

Abbreviations: IDI Intravitreal dexamethasone implant, PBT Peribulbar triamcinolone, logMAR Logarithm of the minimum angle of resolution, $C R T$ Central retinal thickness, CCT Central choroidal thickness, PME Pseudophakic macular edema 


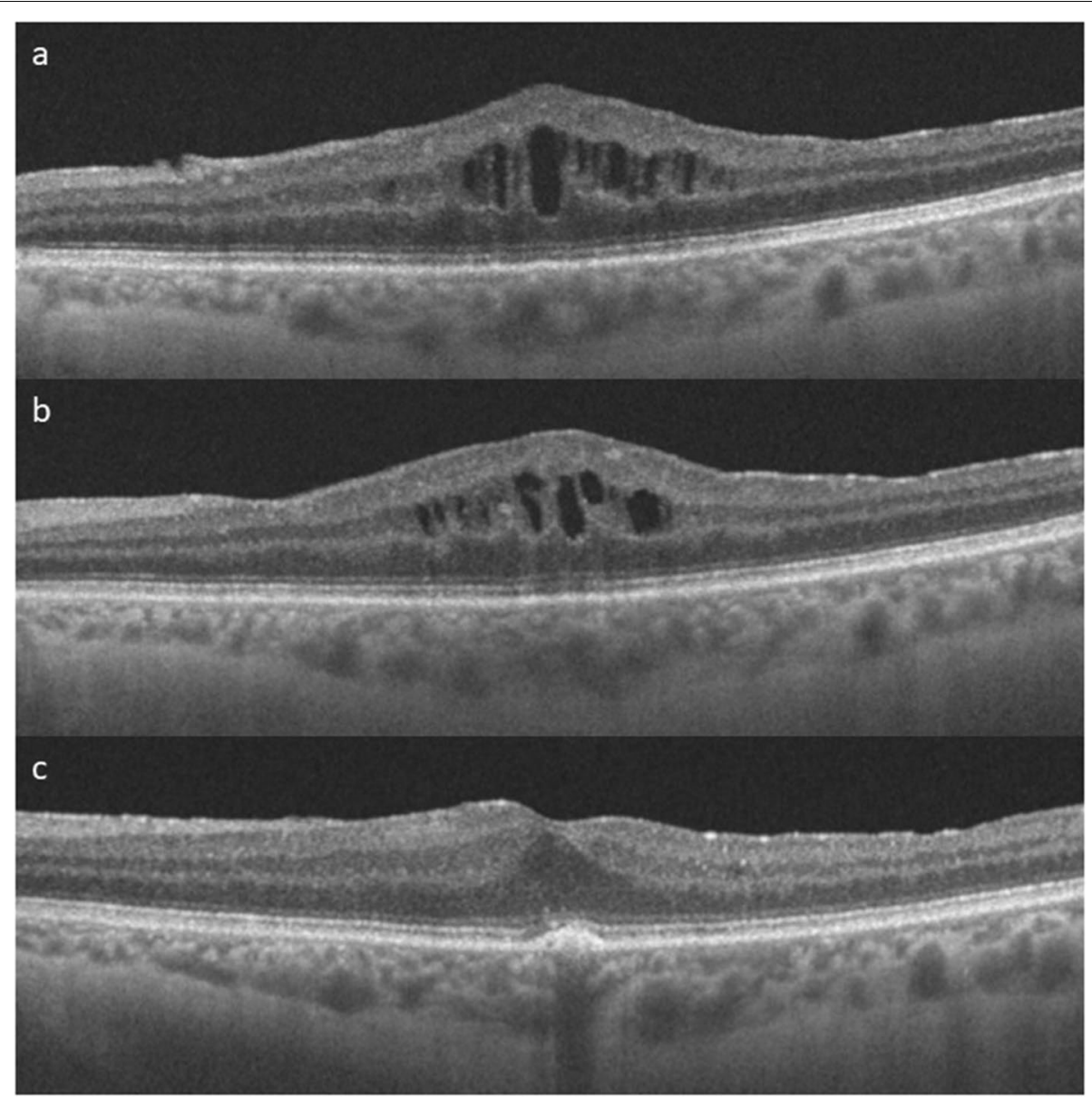

Fig. 2 SS-OCT scans of a patient who received intravitreal dexamethasone implant (IDI) after suboptimal response to topical NSAIDs. a Baseline scan. BCVA: 0.6 logMAR, CRT: $483 \mu \mathrm{m}$, CCT: $267 \mu \mathrm{m}$. b Scan after 2 months of topical NSAIDs. BCVA remained unchanged (0.6 logMAR), CRT decreased to $471 \mu \mathrm{m}$, CCT was $269 \mu \mathrm{m}$. Since BCVA had not improved and CRT had improved scarcely and macular edema persisted, IDI was administered. c SS-OCT scan at 12 months post-IDI. BCVA improved to 0.4 logMAR, CRT decreased to $335 \mu \mathrm{m}$, macular edema was resolved, and CCT decreased to $227 \mu \mathrm{m}$. Disruption of external retinal layers can be observed. No drusen were found in funduscopy

\section{Discussion}

The present retrospective study was performed to evaluate the effectiveness of topical NSAIDs and corticosteroids for the treatment of pseudophakic macular edema in real-life clinical practice. In over $80 \%$ of the patients, the macular edema was successfully resolved by following the treatment algorithm described in Fig. 1. Of the 33 cases, 19 (57.6\%) resolved (no intraretinal or subretinal fluid) with topical NSAIDs after 2 to 9 months of treatment. Median BCVA improved significantly from 0.5 to 0.3 and CRT decreased significantly from 435 to $316 \mu \mathrm{m}$. Of the 13 eyes that received second-line corticosteroid treatment, the edema resolved in 9 eyes $(69.2 \%)$, with a non-significant improvement in median BCVA (from 0.7 to 0.4 ), and a significant decrease in CRT (from 492 to $317 \mu \mathrm{m}$ ) and CCT (from 204 to $182 \mu \mathrm{m}$ ). Intravitreal dexamethasone implant was effective even in a case that had not responded previously to topical NSAIDs and peribulbar triamcinolone.

Guclu et al. [22] applied a 3 month course of topical nepafenac $0.1 \%$ four times a day in 30 eyes with PME, reporting a significant improvement in ETDRS BCVA from 20.9 to 32.9 letters (logMAR scale: approximately 1.3 to 1.1) and a significant decrease in CRT from 501.2 to $364.9 \mu \mathrm{m}$ after 6 months. However, the resolution rate of macular edema was not reported.

Yüksel et al. [23] applied a 12 week course of topical nepafenac $0.1 \%$ three times a day in 24 eyes with PME, reporting a significant $\operatorname{logMAR}$ BCVA improvement from 0.84 to 0.37 , and a significant decrease in CRT from 483.7 to $278.0 \mu \mathrm{m}$ after 6 months. The edema resolved in all patients.

In our study, 33 eyes received once-daily topical nepafenac $0.3 \%$ for at least 2 months. Median BCVA improved significantly from 0.5 to 0.3 and CRT decreased 
significantly from 435 to $316 \mu \mathrm{m}$. The resolution rate of macular edema was $57.6 \%$.

Bellocq et al. [13] applied intravitreal dexamethasone implant in 58 eyes with PME. Of these, $14 \%$ were treatment-naïve, while the others had received previous treatments (topical NSAIDs, oral acetazolamide, intravitreal or subconjunctival triamcinolone, intravitreal antiVEGF). The study included patients with macular edema after cataract and several other types of surgery. Twelve months after a mean of 1.7 injections, the ETDRS BCVA improved significantly from 58.5 to 71 letters (logMAR scale: approximately 0.55 to 0.3 ) and CRT decreased significantly from 518.3 to $342.7 \mu \mathrm{m}$. Intraocular pressure $>25 \mathrm{mmHg}$ was observed in $6.2 \%$ of the patients, but no filtering surgery was required. The specific resolution rate of macular edema for PME at 12 months was not reported. Abdolrahimzadeh et al. [15] applied intravitreal dexamethasone implant in 10 eyes with PME after uncomplicated phacoemulsification. The patients were unresponsive to topical steroids and NSAIDs. Five patients showed recurrence after one intravitreal dexamethasone implant and they received a second implant at month 7. After a twelve-month follow-up, the ETDRS BCVA improved significantly from 62 to 79 letters and CRT decreased significantly from 622 to $282 \mu \mathrm{m}$. Two patients were excluded from the study, and intraocular pressure remained stable during the follow-up. Furino et al. [16] applied a single injection of intravitreal dexamethasone implant in 11 eyes with PME after uncomplicated phacoemulsification. Six months later, BCVA improved significantly from $20 / 40$ to $20 / 22$ and CRT decreased significantly from 462 to $276 \mu \mathrm{m}$. Intraocular pressure did not increase significantly during the follow-up.

Erden et al. [12] applied a single injection of $40 \mathrm{mg}$ subtenon triamcinolone in 21 treatment-naïve eyes diagnosed with PME. In that study, logMAR BCVA improved significantly from 0.71 to 0.24 and CRT decreased significantly from 431 to $299 \mu \mathrm{m}$ after 6 months. The edema resolved in $90 \%$ of patients. Although the intraocular pressure increased slightly, this increase was not statistically significant.

In our study, 13 eyes received second-line corticosteroid treatment (intravitreal dexamethasone implant or peribulbar triamcinolone) after a course of at least 2 months of topical NSAIDs. The edema resolved in 9 eyes (69.2\%), with a non-significant improvement in median BCVA (from 0.7 to 0.4), and a significant decrease in CRT (from 492 to $317 \mu \mathrm{m}$ ).

In our series, macular edema resolved in $84.8 \%$ of 33 eyes treated in accordance with our treatment algorithm (Fig. 1). To our knowledge, this is the first study of PME to describe a specific treatment algorithm and the success rate. Importantly, the effectiveness of corticosteroid treatment in our series could have been underestimated because these second-line treatments were applied in recalcitrant cases that failed to respond to a previous course of topical NSAIDs. In addition, in these cases, the PME had been present for an extended period of time, and the duration of PME may hinder fluid resolution and recovery of BCVA [10, 24]. In this regard, most of the patients treated with corticosteroids showed some degree of disruption of the external retinal layers on SSOCT, which could explain why BCVA did not improve significantly (despite a clear trend towards improvement) even though both retinal and choroidal thicknesses decreased and the edema resolved in most of these cases. Considering these results, we can hypothesize that could be a very effective first-line option for PME, but we cannot confirm this supposition based on our study.

It is worth noting that we administered triamcinolone through the peribulbar route whereas other studies have used the subtenon route $[12,23]$. As a result, it is difficult to directly compare our findings in these patients with other studies. To our knowledge, these two routes of administration have not been previously compared in PME.

The effectiveness of topical NSAIDs could have been underestimated in our study given that results were evaluated after an initial two-month course of treatment (although treatment was continued if some improvement was noted). By comparison, most studies that have evaluated the effectiveness of topical NSAIDs in PME have administered a three-month course of treatment [22, 23].

To date, no randomized trials have been performed to assess the optimal therapeutic protocol for PME. However, our treatment algorithm is a common approach to the management of PME $[9,10]$. Since all of the patients were treated at the same ophthalmology department, the clinical management protocol was the same in all cases, performed in accordance with common criteria. This homogeneous management approach further strengthens the internal validity of our study.

Compared to other studies, the present case series is relatively large, particularly considering the single-institutional nature of the study $[11,12,15,16,18,19]$.

In addition, this is the first study to evaluate choroidal thickness in PME using SS-OCT. Other studies have previously evaluated choroidal thickness in PME using spectral domain OCT (SS-OCT) [25-28]. However, to the best of our knowledge, our study is the first one to evaluate choroidal thickness in PME using Swept-Source OCT. SS-OCT devices use longer wavelengths than spectral domain ones $(1050 \mathrm{~nm}$ versus $840 \mathrm{~nm})$. As a result, they experience less light scattering on the choroid and produce more precise images of the choroid. Besides, in 
our study we have utilized the application of the Triton SS-OCT to define automatically the limits of the choroid, while SD-OCT devices need the operator to manually establish the limits of the choroid in every case. This fact could cause a subjective bias when using SD-OCT to measure choroidal thicknesses, while SS-OCT has proved to be highly reproducible [29-31].

Importantly, we found that CCT decreased significantly after corticosteroid treatment, a finding that appears to support the hypothesis regarding the role of inflammation in PME, which may lead to the disruption of the blood-retina barrier and thus increased vascular permeability and fluid accumulation [7, 8]. Interestingly, this decrease in choroidal thickness was not evidenced in patients treated with topical NSAIDs, potentially due to the greater effectiveness of intravitreal dexamethasone implant [22]. Although peribulbar triamcinolone can resolve PME in cases that fail to respond to topical NSAIDs, one study found no statistically significant difference between the two treatments in terms of CRT in patients with PME [23]. The changes in CCT could shed light on the pathophysiology of PME and guide new treatments and future management of PME, if the association between inflammation and PME is confirmed. The decrease in CCT that we observed in eyes treated with corticosteroids could indicate an association between PME and the pachychoroid spectrum, with choroidal thickness being elevated in PME as occurs in central serous chorioretinopathy or aneurismal type 1 neovascularization [32], but further studies are needed to verify this hypothesis.

\section{Study limitations}

One limitation of this study is the sample size (33 eyes). Although this is larger than many of the studies on PME carried out to date, it is still insufficient to draw any definitive conclusions. Another potential limitation is the evaluation of the corticosteroid treatment, as corticosteroids were only administered in cases refractory to topical NSAIDs after several months of treatment. This could have negatively influenced the effectiveness of corticosteroid treatment in terms of resolution of the edema and final BCVA compared to upfront, first-line treatment with corticosteroids. However, this treatment sequence is widely used in ophthalmology to treat PME because NSAIDs are less invasive and have fewer side effects.

\section{Conclusion}

In this series, topical NSAIDs were an effective treatment for PME, successfully resolving the edema in more than half of the patients. In eyes refractory to topical NSAIDs, corticosteroid treatment (intravitreal dexamethasone implant and peribulbar triamcinolone) were both effective second-line options, yielding good results in more than two-thirds of recalcitrant cases. Overall, the treatment algorithm described here was successful in nearly $85 \%$ of cases. Additional studies, preferably prospective, are needed to confirm these results.

\section{Acknowledgements \\ Not applicable.}

\section{Authors' contributions}

All the authors declare that they have contributed to this submission according to the authorship guidelines. The author(s) read and approved the final manuscript.

\section{Funding}

There are no sources of funding.

\section{Availability of data and materials}

The datasets used and/or analysed during the current study are available from the corresponding author on reasonable request.

\section{Declarations}

Ethics approval and consent to participate

The Clinical Research Ethics Committee of the Hospital Universitari de Bellvitge has approved this project with ref. PR091/20. This Committee is organized and operates in compliance with applicable regulatory requirements and ICH GCP (International Conference on Harmonization Guidelines on Good Clinical Practice).

This is an observational retrospective study and all the data were obtained from the medical records. No identifying data is shown. The need for informed consent was waived by the Clinical Research Ethics Committee of the Hospital Universitari de Bellvitge.

\section{Consent for publication}

Not applicable.

\section{Competing interests}

The authors declare that they have no competing interests.

Received: 9 May 2021 Accepted: 28 September 2021

Published online: 05 November 2021

References

1. Irvine SR. A newly defined vitreous syndrome following cataract surgery. Am J Ophthalmol. 1953;36:599-619.

2. Gass JD, Norton EW. Fluorescein studies of patients with macular edema and papilledema following cataract extraction. Trans Am Ophthalmol Soc. 1966;64:232-49.

3. Kusbeci T, Eryigit L, Yavas G, Inan UU. Evaluation of cystoid macular edema using optical coherence tomography and fundus fluorescein angiography after uncomplicated phacoemulsification surgery. Curr Eye Res. 2012 Apr;37(4):327-33.

4. Bellocq D, Mathis T, Voirin N, Bentaleb ZM, Sallit R, Denis P, et al. Incidence of Irvine Gass syndrome after phacoemulsification with spectraldomain optical coherence tomography. Ocul Immunol Inflamm. 2019;27(8):1224-31.

5. Daien V, Korobelnik J-F, Delcourt C, Cougnard-Gregoire A, Delyfer MN, Bron AM, et al. French medical-administrative database for epidemiology and safety in ophthalmology (EPISAFE): the EPISAFE collaboration program in cataract surgery. Ophthalmic Res. 2017;58(2):67-73.

6. Henderson BA, Kim JY, Ament CS, Ferrufino-Ponce ZK, Grabowska A, Cremers SL. Clinical pseudophakic cystoid macular edema. Risk factors for development and duration after treatment. J Cataract Refract Surg. 2007;33(9):1550-8. 
7. Flach AJ. The incidence, pathogenesis and treatment of cystoid macular edema following cataract surgery. Trans Am Ophthalmol Soc. 1998;96:557-634.

8. $\mathrm{Xu} \mathrm{H}$, Chen M, Forrester JV, Lois N. Cataract surgery induces retinal proinflammatory gene expression and protein secretion. Invest Ophthalmol Vis Sci. 2011;52(1):249-55.

9. Kodjikian L, Bellocq D, Bodaghi B. Management of Irvine-Gass syndrome. J Fr Ophtalmol. 2017;40(9):788-92.

10. Guo S, Patel S, Baumrind B, Johnson K, Levinsohn D, Marcus E, et al. Management of pseudophakic cystoid macular edema. Surv Ophthalmol. 2015;60(2):123-37.

11. Mayer WJ, Kurz S, Wolf A, Kook D, Kreutzer T, Kampik A, et al. Dexamethasone implant as an effective treatment option for macular edema due to Irvine-Gass syndrome. J Cataract Refract Surg. 2015;41(9):1954-61.

12. Erden B, Çakır A, Aslan AC, Bölükbaşi S, Elçioğlu MN. The efficacy of posterior subtenon triamcinolone acetonide injection in treatment of Irvine-Gass syndrome. Ocul Immunol Inflamm. 2019;27(8):1235-41.

13. Bellocq D, Pierre-Kahn V, Matonti F, Burillon C, Voirin N, Dot C, et al. Effectiveness and safety of dexamethasone implants for postsurgical macular oedema including Irvine-Gass syndrome: the EPISODIC-2 study. Br J Ophthalmol. 2017;101(3):333-41.

14. Williams GA, Haller JA, Kuppermann BD, Blumenkranz MS, Weinberg DV, Chou C, et al. Study group. Dexamethasone posterior-segment drug delivery system in the treatment of macular edema resulting from uveitis or Irvine-Gass syndrome. Am J Ophthalmology. 2009;147(6):1048-54.

15. Abdolrahimzadeh S, Fenicia V, Enrici MM, Plateroti P, Cianfrone D, Recupero SM. Twelve-month results of a single or multiple dexamethasone intravitreal implant for macular edema following uncomplicated phacoemulsification. Biomed Res Int. 2015;2015:362564.

16. Furino C, Boscia F, Recchimurzo N, Sborgia C, Alessio G. Intravitreal dexamethasone implant for macular edema following uncomplicated phacoemulsification. European J Ophthalmol May-Jun. 2014;24(3):387-91.

17. Arevalo JF, Maia M, Garcia-Amaris RA, Roca JA, Sanchez JG, Berrocal $\mathrm{MH}$, et al. Pan-American collaborative retina study group. Intravitrea bevacizumab for refractory pseudophakic cystoid macular edema: the Pan-American collaborative retina study group results. Ophthalmology. 2009;116(8):1481-7.

18. Spitzer MS, Ziemssen F, Yoeruek E, Petermeier K, Aisenbrey S, Szurman P. Efficacy of intravitreal bevacizumab in treating postoperative pseudophakic cystoid macular edema. J Cataract Refract Surg. 2008;34(1):70-5.

19. Wu L, Arevalo JF, Hernandez-Bogantes E, Roca JA. Intravitreal infliximab for refractory pseudophakic cystoid macular edema: results of the Pan-American collaborative retina study group. Int Ophthalmol. 2012;32(3):235-43.

20. Giganti M, Beer PM, Lemanski N, Hartman C, Schartman J, Falk N. Adverse events after intravitreal infliximab (Remicade). Retina. 2010;30(1):71-80.
21. Bellocq D, Korobelnik JF, Burillon C, Voirin N, Dot C, Souied E, et al. Effectiveness and safety of dexamethasone implants for post-surgical macular oedema including Irvine-Gass syndrome: the EPISODIC study. $\mathrm{Br}$ J Ophthalmol. 2015;99(7):979-83.

22. Guclu H, Pelitli GV. Comparison of topical nepafenac $0.1 \%$ with intravitreal dexamethasone implant for the treatment of Irvine-Gass syndrome. Int J Ophthalmol. 2019;12(2):258-67.

23. Yüksel B, Uzunel UD, Kerci SG, Sağban L, Küsbeci T, Örsel T. Comparison of Subtenon triamcinolone Acetonide injection with topical Nepafenac for the treatment of Pseudophakic cystoid macular edema. Ocul Immunol Inflamm. 2017;25(4):513-9.

24. Teja S, Sawatzky L, Wiens T, Maberley D, Ma P. Ozurdex for refractory macular edema secondary to diabetes, vein occlusion, uveitis and pseudophakia. Can J Ophthalmol. 2019;54(5):540-7.

25. Pierru A, Carles M, Gastaud P, Baillif S. Measurement of subfoveal choroidal thickness after cataract surgery in enhanced depth imaging optical coherence tomography. Invest Ophthalmol Vis Sci. 2014;55(8):4967-74.

26. Odrobina D, LaudaŃska-Olszewska I. Choroidal thickness in clinically significant pseudophakic cystoid macular edema. Retina. 2015;35(1):136-40.

27. Fleissig E, Cohen S, Iglicki M, Goldstein M, Zur D. Changes in choroidal thickness in clinically significant pseudophakic cystoid macular edema. Retina. 2018;38(8):1629-35.

28. Jiang H, Li Z, Sun R, Liu D, Liu N. Subfoveal Choroidal and macular thickness changes after phacoemulsification using enhanced depth imaging optical coherence tomography. Ophthalmic Res. 2018;60(4):243-9.

29. Copete S, Flores-Moreno I, Montero JA, Duker JS, Ruiz-Moreno JM. Direct comparison of spectral-domain and swept-source OCT in the measurement of choroidal thickness in normal eyes. Br J Ophthalmol. 2014;98:334-8.

30. Obis J, Garcia-Martin E, Orduna E, Vilades E, Alarcia R, Rodrigo MJ, et al. Reproducibility of retinal and choroidal measurements using sweptsource optical coherence tomography in patients with Parkinson's disease. Arq Bras Oftalmol. 2020;83(1):19-27.

31. Pinilla I, Sanchez-Cano A, Insa G, Bartolomé I, Perdices L, Orduna-Hospital E. Choroidal differences between spectral and swept-source domain technologies. Curr Eye Res. 2021;46(2):239-47.

32. Cheung CMG, Lee WK, Koizumi H, Dansingani K, Lai TYY, Freund KB. Pachychoroid disease. Eye (Lond). 2019;33(1):14-33.

\section{Publisher's Note}

Springer Nature remains neutral with regard to jurisdictional claims in published maps and institutional affiliations.
Ready to submit your research? Choose BMC and benefit from:

- fast, convenient online submission

- thorough peer review by experienced researchers in your field

- rapid publication on acceptance

- support for research data, including large and complex data types

- gold Open Access which fosters wider collaboration and increased citations

- maximum visibility for your research: over $100 \mathrm{M}$ website views per year

At BMC, research is always in progress.

Learn more biomedcentral.com/submissions 